\title{
Screening for Olfactory Dysfunction in COVID-19 Patients Using Quick Smell Identification Test
}

\author{
Hussain Albaharna ${ }^{1}$, Alya Alhajjaj ${ }^{1}$, Hussain Al Shabeb ${ }^{1}$, Dhuha Alghanim ${ }^{1}$, Fatimah \\ Alkhabbaz $^{1}$, and Jumana AlJishi ${ }^{1}$ \\ ${ }^{1}$ Qatif Central Hospital
}

November 6, 2020

\begin{abstract}
Intro: To determine the prevalence of OD in the confirmed case with COVID-19 among our population using quick smell identification test (Q-SIT) as screening tool. Methods: Cross- sectional study carried out in Qatif area - Saudi Arabia among adult hospitalized patient with confirm COVID-19 during the period between May and July, 2020. All adults confirmed COVID19 patients were interviewed for history of current disease and associated symptoms as well as performing Q-SIT. Participants who had history of olfactory dysfunction, and critical cases required ICU admission were excluded. Results: The prevalence of OD among COVID-19 cases was (16.3\%) in our population using Q-SIT compared to (27.4\%) for self-reported symptom. Females were having higher prevalence in compare to males $(30.5 \%$ and $11.1 \%)$ respectively; which was statistically significant $(\mathrm{P}<0.001)$. The patients reported higher prevalence of ageusia $(31.9 \%)$ with significant association with OD $(\mathrm{P}<0.001)$. Q-SIT showed high positive and negative predictive value in detecting OD among patients with COVID-19 (84\% and $93 \%$ respectively). Conclusion: Q-SIT is a useful, validated and easy to apply tool for screening OD among patients with COVID-19. Some patients presented solely with this symptom which can occurs unnoticed in COVID-19 patients, and there for required objective test for detection.
\end{abstract}

Title: Screening for Olfactory Dysfunction in COVID-19 Patients Using Quick Smell Identification Test: Cross-sectional study

\section{Abstract}

Objective: To determine the prevalence of OD in the confirmed case with COVID-19 among our population using Quick Smell Identification Test (Q-SIT) as screening tool.

Design: Cross- sectional study carried out in Qatif area - Saudi Arabia among adult hospitalized patient with confirm COVID-19 during the period between May and July, 2020.

Setting: All patients were interviewed for demographic data, history of the current disease and associated symptoms as well as performing Q-SIT.

Participants: All confirmed COVID-19 patients, both male and female, and adults aged 18 years or above were included. Participants who had history of olfactory dysfunction, and critical cases required ICU admission were excluded. The sample size was estimated using the CDC tool (Epi Info[?]) to be 260 subjects.

Main Outcome Measures: The prevalence of OD among COVID-19 using Q-SIT as screening tool.

Results: The prevalence of OD among COVID-19 cases was (16.3\%) in our population using Q-SIT compared to $(27.4 \%)$ for self-reported symptom. Females were having higher prevalence in compare to males $(30.5 \%$ and $11.1 \%)$ respectively; which was statistically significant $(\mathrm{P}<0.001)$. The patients reported higher 
prevalence of ageusia $(31.9 \%)$ with significant association with OD $(\mathrm{P}<0.001)$. Q-SIT showed high positive and negative predictive value in detecting OD among patients with COVID-19 (84\% and 93\% respectively).

Conclusions: Q-SIT is a useful, validated and easy to apply tool for screening OD among patients with COVID-19. Some patients presented solely with this symptom which can occurs unnoticed in COVID-19 patients, and there for required objective test for detection.

Key words: COVID-19, Olfaction Disorders, Odor, Prevalence, Smell test

\section{Key points:}

- Olfactory dysfunction can be the only presenting symptom of COVID-19. Hens, finding a validated screening tool for OD is valuable.

- Q-SIT is a Three-item microencapsulated odor identification psychophysical (objective) test.

- The prevalence of OD among COVID-19 cases was (16.3\%) in our population using Q-SIT compared to $(27.4 \%)$ for self-reported symptom.

- Q-SIT showed high positive and negative predictive value in detecting OD among patients with COVID19 (84\% and $93 \%$ respectively).

- Q-SIT is a useful, validated and easy to apply tool for screening OD among patients with COVID-19.

\section{Introduction}

With the emergence of Coronavirus disease 2019 (COVID-19) outbreak on December $2019^{1}$, many researches have been published about its transmission, diagnosis, clinical presentation and management. Its presentation varies widely from mild to severe symptoms including severs pneumonia. Though the main reported COVID-19 symptoms include fever, headache, gastrointestinal symptoms, and respiratory symptoms. ${ }^{2}$ Upper respiratory symptoms where not uncommon such as sore throat, rhinorrhea, complete or partial loss of smell (olfactory dysfunction-OD). ${ }^{3}$ Post viral Olfactory dysfunction (PVOD) Is caused by different viruses including Rhinoviruses, coronaviruses, parainfluenza viruses, and Epstein-Barr viruses. ${ }^{4}$ Many preliminary reports have suggested that smell and taste loss are potential early symptom or subclinical markers of COVID-19 infection. Several cross-sectional studies from many countries such as Iran, United Kingdom, Italy, Spain, Germany, European countries, France, and united states have been published about OD prevalence in COVID-19 patients. ${ }^{5,6}$ The incidence of OD in COVID-19 patients differs widely between these cross-sectional studies. ranging from 33.9 to $68 \% .^{5}$

A study from Spain using a self-reported questionnaire only without a validated olfactory test, found that the incidence rate of OD in COVID-19 patients was significantly more than OD in influenza patients (39.2\% vs12.5\%). ${ }^{7}$ Olfactory tests are categorized in to 3 types: subjective, psychophysical, and electorophysiological studies. Subjective testing can be performed through self-reporting method or as a part of quality of life outcome questionnaire eg. Sinonasal outcome test-22 (SNOT-22). Many tests have been utilized to assess the olfaction function objectively. These are the psychophysical tests which measure some or all the three olfactory parameters: the threshold, discrimination and identification. While subjective and psychophysical tests are used in most clinical and research, the electrophysiological studies like electroencephalography (EEG) and electro-olfactography (EOG) are having limited clinical use and mainly performed for medicolegal issues. $^{8}$

One observational study from Saudi Arabia, found that self- reported loss of taste and smell was the most common presentation (47.5\%.) in mild symptomatic COVID-19 patients. ${ }^{9}$ Objective (psychophysical test) has been available in few studies only, though it is considered to be the gold standard for diagnosis of OD..$^{2,10,11}$ Moein et al reported that only $35 \%$ of their subjects were aware about their smell problem before doing objective test which indicate that self-reporting of the symptoms may be not enough and the incidence rate of OD is much higher than reported by the previous studies. ${ }^{10}$

This study aims to use Quick Smell Identification Test (Q-SIT) as screening tool to assess the prevalence of olfactory dysfunction in patients with confirmed COVID-19 infection in Qatif area, Eastern province, Saudi Arabia. 


\section{Materials and Methods}

\section{Study Design and Participants}

This cross-sectional study was conducted in Qatif area, Saudi Arabia, during the period between May and July, 2020. Majority of our patients were severe cases hospitalized in Qatif central hospital wards, with positive results on reverse transcriptase polymerase chain reaction (RT-PCR) testing of COVID 19. Patients considered mild to moderate COVID-19, when there was no O2 requirements, no evidence of pneumonia but with other symptoms of covid19 e.g. fever. While Severe cases defined as the presence of [?] 1 of the following symptoms: Respiratory rate [?]30/min (adults), blood oxygen saturation [?]93\%, $\mathrm{PaO} 2 / \mathrm{FiO} 2$ ratio $<300$, lung infiltrates $>50 \%$ of the lung field within $24-48$ hours. $^{12}$

Our inclusion criteria included all confirmed COVID-19 patients (Saudi and nonSaudi), both male and female, and adults aged 18 years or above. Participants who had history of olfactory dysfunction, their age were below 18 years and critical COVID-19 pneumonia required intensive care unit admission were also excluded.

\section{Sampling}

According to the Saudi ministry of health, the estimated number of confirmed COVID-19 cases in the Qatif area during the study period was around 7000 cases. ${ }^{13}$ The sample size was calculated using the Centers for Disease Control and Prevention (CDC) tool (Epi Info[?] For Windows version 7.2). For confidence level of $90 \%$ the estimated sample size was 260 subjects.

\section{Data Collection}

We conducted a face-to-face interview with the participants. We Collected basic demographic, epidemiological and clinical data of hospitalized patients with COVID-19. Data on comorbidities, past medical and surgical history, olfactory dysfunction data were obtained. Quick- Smell Identification Test was performed in all patients.

\section{Olfactory Testing}

Olfactory function screening was done through Q-SIT. This is a Three-item microencapsulated odor identification test of standardized odors with five multiple choice options, one is "none/other" (Figure 1). Question one is testing chocolate odor, while the second is testing banana odor and third is for smoke odor. ${ }^{14}$

This test was chosen in particular because it is tear-off card test (disposable) so there is no concern about contamination and transmission of disease form COVID-19 patients. Moreover, it is fast and can be administered with in less than 1 minute. ${ }^{15}$ The test was validated against University of Pennsylvania Smell Identification Test (UPSIT). Though we disproved the validity of UPSIT in previous publication on our population, the three odorants used in Q-SIT are validated and accurately identified by our population. ${ }^{16}$ Cutoff point on one wrong answer gives better sensitivity and specificity with negative and positive predictive value of $98 \%, 43 \%$ respectively for detecting anosmia. ${ }^{14}$ Accordingly, we considered cutoff score of [?] 2 to be normal test and cutoff score of [?] 1 to be abnormal test for anosmia.

\section{Statistical Analysis}

Data were entered and analyzed using SPSS (version 25). The mean and standard deviation were calculated for numerical variables while count and percentages for categorical variables. Chi-square and Fisher's exact test were used to test for significant difference and P-value [?] 0.05 considered statistically significant.

\section{Reporting Guideline}

We have followed The Strengthening the Reporting of Observational Studies in Epidemiology (STROBE) Statement to write this study.

\section{Ethical Approval}


Informed written consent was obtained from each patient. The study was approved by the Institutional Research Ethic Committee in $<$ Blinded for review $>$. Tests were performed with appropriate personal protective

equipment to ensure the examiner's safety.

\section{Data availability statement}

The data that support the findings of this study are available on request from the corresponding author. The data are not publicly available due to privacy or ethical restrictions.

\section{Results}

\section{The patients' Characteristics}

We interviewed a total of 275 patients with PCR- confirmed COVID-19 infection. Five patients were excluded (three patients aged less than 18 years, two patients reported previous history of OD). Of 270 patients, 250 patients $(88 \%)$ were hospitalized with severe COVID-19 pneumonia. Only 20 patients $(12 \%)$ reported mild symptoms and they were not hospitalized. The majority were in the age group 36-45 (30\%). The median age was 43 years, 198 (73.3\%) were male. Most of our participants 177 (65.6\%) were Saudi (Table 1).

\section{Clinical Features and Past History}

Table 2 shows the presenting symptoms and comorbid illness of the participants. Fever was the most prevalent symptom in 218 patients (80.7\%) followed by cough in 197 patients (73\%). A total of 74 patients $(27.4 \%)$ reported loss of smell; being the first symptom in $7 \%$. Furthermore, $86(31.9 \%)$ reported ageusia. The median duration for anosmia was 4 (1-15) days where as the median duration for ageusia was 5 (1-15) days. A total of 21 patients $(7.8 \%)$ reported past history of chronic rhinosinusitis or allergic rhinitis without OD.

\section{Olfactory Tests}

Q-SIT was used to screen all included patients regardless of the presence or absence of OD. Table 1 shows the Q-SIT scores of all participants. The prevalence of OD using Q-SIT was 16.3\% (44 participants). Female were having higher prevalence in comparison to males $(30.5 \%$ and $11.1 \%)$ respectively; which was statistically significant $(\mathrm{P}<0.001)$. On the other hand, our results showed no significant association between age, nationality, or comorbid illness including chronic rhinosinusitis with self-reported anosmia or abnormal Q-SIT (Table 1). Furthermore, no significant association was found between anosmia and nasal blockage, postnasal drip, and rhinorrhea with olfactory dysfunction.

Among patients with abnormal Q-SIT, 37 patients (84\%) subjectively reported OD at the time of the test. The recognition rate to question 1 (chocolate odor) was better than other two odors for patients with OD (Fig 2). Both ageusia and abnormal Q-SIT were present in $38.4 \%$ of patients with statistical significance (P $<0.001$; Table 3).

In patients with anosmia at the time of Q-SIT administration, $75.5 \%$ of the participants had abnormal Q-SIT when cutoff score [?] 2 was used; whereas $69.8 \%$ of the participants had abnormal Q-SIT when cutoff score [?] 1 was used (table 5b,5a). On the other hand, in patients without anosmia at the time of Q-SIT administration, $30.4 \%$ of the participants had abnormal Q-SIT when cutoff score [?] 2 was used; whereas only $3.2 \%$ of the participants had abnormal Q-SIT when cutoff score [?] 1 was used. (Table 4).

\section{Discussion}

In our study, we found that the estimated prevalence of self- reported OD in our sample was $27.4 \%$ while the prevalence of abnormal olfactory test was $16.3 \%$ which is lower than what was reported by most recent studies including studies using standard olfactory tests. ${ }^{5}$ Furthermore, we found that OD was more prevalent in females with confirmed COVID-19 infection which is similar to most published studies. 
The low prevalence of OD in our study in comparison with other studies can be explained by the fact that the majority of our patients were hospitalized with severe COVID-19 pneumonia. So, the low prevalence could be explained by the delayed testing which led to the partial recovery of the olfactory dysfunction. While other studies examined patients with mild- moderate COVID-19 disease in their early stage. Moreover, psychophysical tests such as the Q-SIT evaluate one's sense of smell at a specific point in time, which could lead to an underestimation of the prevalence of OD. Jerome R. et al. showed that about $38.3 \%$ of patients with self-reported sudden-onset olfactory dysfunction found to be normosmic by the psychophysical Sniffin' Sticks test. we recorded similar finding of $30.2 \%$ having normal Q-SIT while they subjectively reported OD. ${ }^{17}$

When we measured the association between the Q-SIT and subjectively reported OD at the time of test, we found better positive predictive value and negative predictive value on a cutoff score [?] 1 ( $84 \%$ and $93 \%$ respectively) in compare to the cutoff score [?] 2. (Table 5) For that we have used the former cutoff in all previously mentioned calculations.

This study showed no significant association between olfactory dysfunction and nasal symptoms. This is supporting the hypothesis of direct invasion of the olfactory neurons by SARS-CoV-2 as the virus could be replicated in neural cell line U251 in vitro. ${ }^{18}$ That is against other hypothesis of olfactory cleft blockage due to inflammation or inflammatory cytokines affecting olfactory neural mucosa. ${ }^{6}$

It was expected to have significant association between OD and abnormal tasting (38.4\%) as retronasal olfaction is the cause of most gustatory impairment. ${ }^{19}$ Moreover, the prevalence of gustatory impairment $(31.8 \%)$ in our sample was higher than olfactory impairment. These data are supported by similar findings of an epidemiological survey conducted in four European countries. ${ }^{20}$ while some studies differentiate between olfactory and gustatory dysfunction, others just report the prevalence of both anosmia and Ageusia as one symptom. ${ }^{7,21,22}$

\section{Strength and limitations}

We have reported very valuable and validated objective tool for screening OD in patient with severe COVID19. This is considered a strength to our study. Moreover, using an objective test avoids the response bias as patients can be influenced by the news that report smell and taste dysfunction in COVID-19 and overreported these symptoms.

On the other hand, this study has some limitations where most of our study population was having moderate to severe COVID 19 symptoms whom required hospitalization; for that mild cases were missed. Finally, duration of the symptoms and recovery rate were not assessed very well in this study.

\section{Conclusion}

Although the fever was considered the most frequent reported sign and symptoms in COVD 19 Patients, OD is one of the confirm symptoms to predict COVID-19 infection. Q-SIT is a useful, validated and easy to apply tool for screening OD specially in the current situation. Some patients have presented solely with this symptom usually in mild cases. Hence, primary physicians and otolaryngologist need to be aware of this putative presentation. Our study shows that anosmia can occurs unnoticed in COVID-19 patients, and there for those patients required objective and quantifiable test for detection.

\section{References:}

1. Zhu N, Zhang D, Wang W, Li X, Yang B, Song J, et al. A novel coronavirus from patients with pneumonia in China, 2019. N Engl J Med. 2020;382(8):727-33.

2. Ottaviano G, Carecchio M, Scarpa B, Marchese-Ragona R. Olfactory and rhinological evaluations in SARS-CoV-2 patients complaining of olfactory loss. Rhinol J [Internet]. 2020 Apr 1;382(18):0-0. Available from: https://www.rhinologyjournal.com/Abstract.php?id=2462 
3. Lovato A, de Filippis C. Clinical Presentation of COVID-19: A Systematic Review Focusing on Upper Airway Symptoms. Ear, Nose Throat J. 2020;

4. Suzuki M, Saito K, Min WP, Vladau C, Toida K, Itoh H, et al. Identification of viruses in patients with postviral olfactory dysfunction. Laryngoscope. 2007;117(2):272-7.

5. Meng X, Deng Y, Dai Z, Meng Z. COVID-19 and anosmia: A review based on upto-date knowledge. Am J Otolaryngol [Internet]. 2020 Sep;41(5):102581. Available from: https://linkinghub.elsevier.com/retrieve/pii/S0196070920302751

6. Chung TWH, Sridhar S, Zhang AJ, Chan KH, Li HL, Wong FKC, et al. Olfactory Dysfunction in Coronavirus Disease 2019 Patients: Observational Cohort Study and Systematic Review. Open Forum Infect Dis. 2020;7(6):1-12.

7. Beltrán-Corbellini, Chico-García JL, Martínez-Poles J, Rodríguez-Jorge F, Natera-Villalba E, GómezCorral J, et al. Acute-onset smell and taste disorders in the context of COVID-19: a pilot multicentre polymerase chain reaction based case-control study. Eur J Neurol. 2020;27(9):1738-41.

8. Hummel T, Whitcroft KL, Andrews P, Altundag A, Cinghi C, Costanzo RM, et al. Position paper on olfactory dysfunction. Rhinology. 2017;54:1-30.

9. Alshami AA, Alattas RA, Anan HF, Qahtani HS Al, Mulhim MA Al, Alahilmi AA, et al. Silent Disease and Loss of Taste and Smell are Common Manifestations of SARS-COV-2 Infection in a Quarantine Facility: First report from Saudi Arabia. medRxiv. 2020;2020.05.13.20100222.

10. Moein ST, Hashemian SMR, Mansourafshar B, Khorram-Tousi A, Tabarsi P, Doty RL. Smell dysfunction: a biomarker for COVID-19. Int Forum Allergy Rhinol. 2020;10(8):944-50.

11. Vaira LA, Salzano G, Petrocelli M, Deiana G, Salzano FA, De Riu G. Validation of a self-administered olfactory and gustatory test for the remotely evaluation of COVID-19 patients in home quarantine. Head Neck. 2020;42(7):1570-6.

12. Care S, X-ray C, X-ray C, X-ray C. Saudi MoH Protocol for Adults Patients Suspected of / Confirmed with COVID-19 Supportive care and antiviral treatment of suspected or confirmed COVID-19 infection Saudi MoH Protocol for Adults Patients Suspected of / Confirmed with COVID-19 Supportive car. 2020;1-6.

13. https://sehhty.com/sa-covid10/ [Internet]. Available from: https://sehhty.com/sa-covid10/

14. Jackman AH, Doty RL. Utility of a three-item smell identification test in detecting olfactory dysfunction. Laryngoscope. 2005;115(12):2209-12.

15. Malaty J, Malaty IAC. Smell and taste disorders in primary care. Am Fam Physician. 2013;88(12):852-9.

16. Albaharna H, Almubaireek H, Upadhyay S, Buohliqah L. Validity of the Arabic version of the University of Pennsylvania smell test on a Saudi population. Pan Arab J Rhinol J. 2017;7:7-10.

17. Lechien JR, Cabaraux P, Chiesa-Estomba CM, Khalife M, Hans S, Calvo-Henriquez C, et al. Objective olfactory evaluation of self-reported loss of smell in a case series of 86 COVID-19 patients. Head Neck. 2020;42(7):1583-90.

18. Chu H, Chan JF-W, Yuen TT-T, Shuai H, Yuan S, Wang Y, et al. Comparative tropism, replication kinetics, and cell damage profiling of SARS-CoV-2 and SARS-CoV with implications for clinical manifestations, transmissibility, and laboratory studies of COVID-19: an observational study. The Lancet Microbe [Internet]. 2020;1(1):e14-23. Available from: http://dx.doi.org/10.1016/S2666-5247(20)30004-5

19. Deems DA, Doty RL, Settle RG, Moore-Gillon V, Shaman P, Mester AF, et al. Smell and Taste Disorders, A Study of 750 Patients From the University of Pennsylvania Smell and Taste Center. Arch Otolaryngol Neck Surg. 1991;117(5):519-28. 
20. Lechien JR, Chiesa-Estomba CM, De Siati DR, Horoi M, Le Bon SD, Rodriguez A, et al. Olfactory and gustatory dysfunctions as a clinical presentation of mild-to-moderate forms of the coronavirus disease (COVID-19): a multicenter European study. Eur Arch Oto-Rhino-Laryngology [Internet]. 2020;277(8):225161. Available from: https://doi.org/10.1007/s00405-020-05965-1

21. Borsetto D, Hopkins C, Philips V, Obholzer R, Tirelli G, Polesel J, et al. Self-reported alteration of sense of smell or taste in patients with COVID-19: a systematic review and meta-analysis on 3563 patients. Rhinology [Internet]. 2020;(July):1-7. Available from: https://doi.org/10.4193/Rhin20.185

22. Spinato G, Fabbris C, Polesel J, Cazzador D, Borsetto D, Hopkins C, et al. Alterations in Smell or Taste in Mildly Symptomatic Outpatients With SARS-CoV-2 Infection. JAMA [Internet]. 2020 May 26;323(20):2089. Available from: https://jamanetwork.com/journals/jama/fullarticle/2765183

Figure 1: sample of the quick smell identification test card. Notice the tear-off slip with three questions on right side. Patient information and the answer key on the left side on the card.

Figure 2: Response rate to different odorants in Q-SIT among included patients.

\begin{tabular}{|c|c|c|c|c|}
\hline $\begin{array}{l}\text { Table 1. Patients } \\
\text { Characteristics }\end{array}$ & $\begin{array}{l}\text { Table 1. Patients } \\
\text { Characteristics }\end{array}$ & & & \\
\hline$\overline{\text { Characteristics }}$ & $\begin{array}{l}\text { All Patients } \\
(\mathbf{n}=\mathbf{2 7 0})\end{array}$ & $\begin{array}{l}\text { Normal Q-SIT } \\
(n=226)\end{array}$ & $\begin{array}{l}\text { Abnormal Q-SIT } \\
(\mathrm{n}=44)\end{array}$ & $P$ value \\
\hline $\begin{array}{l}\text { Demographic } \\
\text { data }\end{array}$ & $\begin{array}{l}\text { Demographic } \\
\text { data }\end{array}$ & & & \\
\hline $\begin{array}{l}\text { Age }(Y) \text { - mean } \\
(\mathrm{SD})\end{array}$ & $43( \pm 12)$ & $44( \pm 12)$ & $39( \pm 11)$ & 0.109 \\
\hline $18-25$ - no. $(\%)$. & $18(6.7)$ & $12(5.3)$ & $6(13.6)$ & \\
\hline 26-35 - no. (\%). & $59(21.9)$ & $47(20.8)$ & $12(27.3)$ & \\
\hline $36-45$ - no. (\%). & $81(30.0)$ & $66(29.2)$ & $15(34.1)$ & \\
\hline $46-55$ - no. $(\%)$ & $69(25.6)$ & $62(27.4)$ & $7(15.9)$ & \\
\hline $56-65$ - no. $(\%)$. & $34(12.6)$ & $30(13.3)$ & $4(9.1)$ & \\
\hline$>65-$ no. $(\%)$ & $9(3.3)$ & $9(4.0)$ & $0(0)$ & \\
\hline Sex & & & & $<0.001$ \\
\hline Male - no. (\%). & $198(73.3)$ & $176(77.9)$ & $22(50.0)$ & \\
\hline Female - no. (\%). & $72(26.7)$ & $50(22.1)$ & $22(50.0)$ & \\
\hline Nationality & & & & 0.795 \\
\hline Saudi - no. (\%). & $177(65.6)$ & $143(63.3)$ & $34(77.3)$ & \\
\hline $\begin{array}{l}\text { Arab, non-Saudi } \\
\text { - no. }(\%) .\end{array}$ & $9(3.3)$ & $7(3.1)$ & $2(4.5)$ & \\
\hline Indian - no. (\%). & $25(9.3)$ & $23(10.2)$ & $2(4.5)$ & \\
\hline $\begin{array}{l}\text { Pakistani - no. } \\
(\%) .\end{array}$ & $19(7.0)$ & $17(7.5)$ & $2(4.5)$ & \\
\hline $\begin{array}{l}\text { Bangladeshi - no. } \\
(\%) \text {. }\end{array}$ & $19(7.0)$ & $16(7.1)$ & $3(6.8)$ & \\
\hline $\begin{array}{l}\text { Filipino - no. } \\
(\%) .\end{array}$ & $14(5.2)$ & $13(5.8)$ & $1(2.3)$ & \\
\hline Others- no. (\%). & $7(2.6)$ & $7(3.1)$ & $0(0)$ & \\
\hline Abbreviations: & Abbreviations: & Abbreviations: & Abbreviations: & Abbreviations: \\
\hline $\begin{array}{l}\text { Q-SIT, quick- } \\
\text { smell }\end{array}$ & $\begin{array}{l}\text { Q-SIT, quick- } \\
\text { smell }\end{array}$ & $\begin{array}{l}\text { Q-SIT, quick- } \\
\text { smell }\end{array}$ & $\begin{array}{l}\text { Q-SIT, quick- } \\
\text { smell }\end{array}$ & $\begin{array}{l}\text { Q-SIT, quick- } \\
\text { smell }\end{array}$ \\
\hline identification & identification & identification & identification & identification \\
\hline Test. & Test. & Test. & Test. & Test. \\
\hline
\end{tabular}


Clinical Features

Partial Anosmia - no. (\%).

Complete Anosmia - no. (\%).

Anosmia as The First symptom - no. (\%).

Ageusia - no. (\%).

Rhinorrhea - no. (\%).

Nasal blockage - no. (\%).

Fever - no. (\%).

Cough - no. (\%).

Sore throat - no. (\%).

SOB - no. (\%).

Diarrhea - no. (\%).

Headache - no. (\%).

Sputum production - no. (\%).

Asthenia - no. (\%).

Loss of appetite - no. (\%).

Arthralgia - no. (\%).

Myalgia - no. (\%).

Abdominal pain - no. (\%).

Nausea - no. (\%).

Vomiting - no. (\%).

Ear pain - no. (\%).

Past History

Chronic sinusitis - no. (\%).

Nasal polyp - no. (\%).

Allergic rhinitis - no. (\%).

DM - no. (\%).

HTN - no. (\%).

COPD - no. (\%).

Asthma - no. (\%).

CKD - no. (\%).

CLD - no. (\%).

CVD - no. (\%).

GERD - no. (\%).

Hypothyroidism - no. (\%).

Depression - no. (\%).

Autoimmune disease - no. (\%).

Sinus surgery - no. (\%)

Smoking - no. (\%)

Abbreviations: Q-SIT: quick- smell identification Test; SOB: Shortness of Breath; DM: diabetes mellitus; HTN: Hypertensi

Table 3: Q-SIT and Ageusia

\begin{tabular}{llll}
\hline & Ageusia & Ageusia & Ageusia \\
Q-SIT & Yes & No & P value \\
Normal - no. (\%). & $53(61.6 \%)$ & $173(94 \%)$ & $<0.001$ \\
Abnormal - no. (\%). & $33(38.4 \%)$ & $11(5.99 \%)$ & $<0.001$
\end{tabular}


Table 4: correlation between subjectively reported smell impairment and Q-SIT using different cutoff point scores

\section{Q-SIT}

Cutoff score [?] 2

Normal score -no. (\%).

Abnormal score -no. (\%).

Cutoff score [?] 1

Normal score -no. (\%).

Abnormal score -no. (\%).

Total

$\begin{array}{ll}\begin{array}{l}\text { Anosmia During Smell Test } \\ \text { Yes }\end{array} & \begin{array}{l}\text { Anosmia During Smell Test } \\ \text { No }\end{array} \\ \text { Cutoff score [?] } 2 & \text { Cutoff score [?] } 2 \\ 13(24.5) & 151(69.6) \\ 40(75.5) & 66(30.4) \\ \text { Cutoff score [?] } 1 & \text { Cutoff score [?] } 1 \\ 16(30.2) & 210(96.8) \\ 37(69.8) & 7(3.2) \\ 53 & 217\end{array}$

Anosmia During Smell $P$ value

Cutoff score [?] 2

$<0.001$

$<0.001$

Cutoff score [?] 1

$<0.001$

$<0.001$

270

Table 5: Sensitivity, Specificity, Positive Predictive Value (PPV), and Negative Predictive Value (NPV) Q-SIT using different cutoff point scores in relation to subjectively reported smell impairment.

\begin{tabular}{|c|c|c|c|c|}
\hline Q-SIT & $\mathrm{Se}$ & $\mathrm{Sp}$ & PPV & NP \\
\hline Abl & 75 & 69 & $37.7 \%$ & $92 \%$ \\
\hline Abnormal score with cutoff score [?] 1 & $69.9 \%$ & $96.8 \%$ & $84 \%$ & $93 \%$ \\
\hline
\end{tabular}

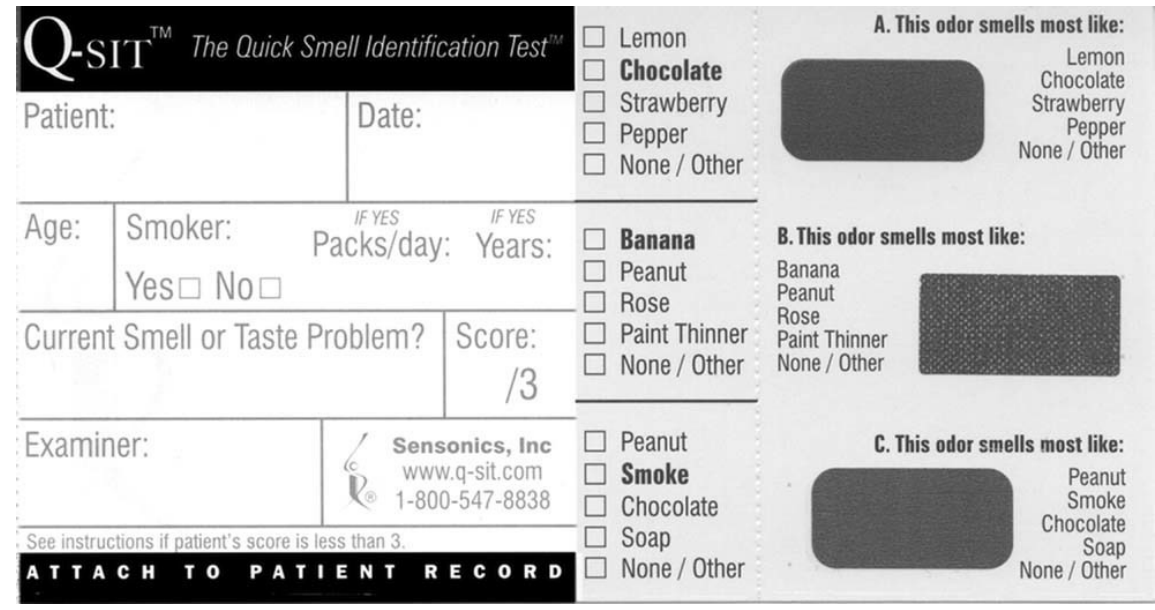




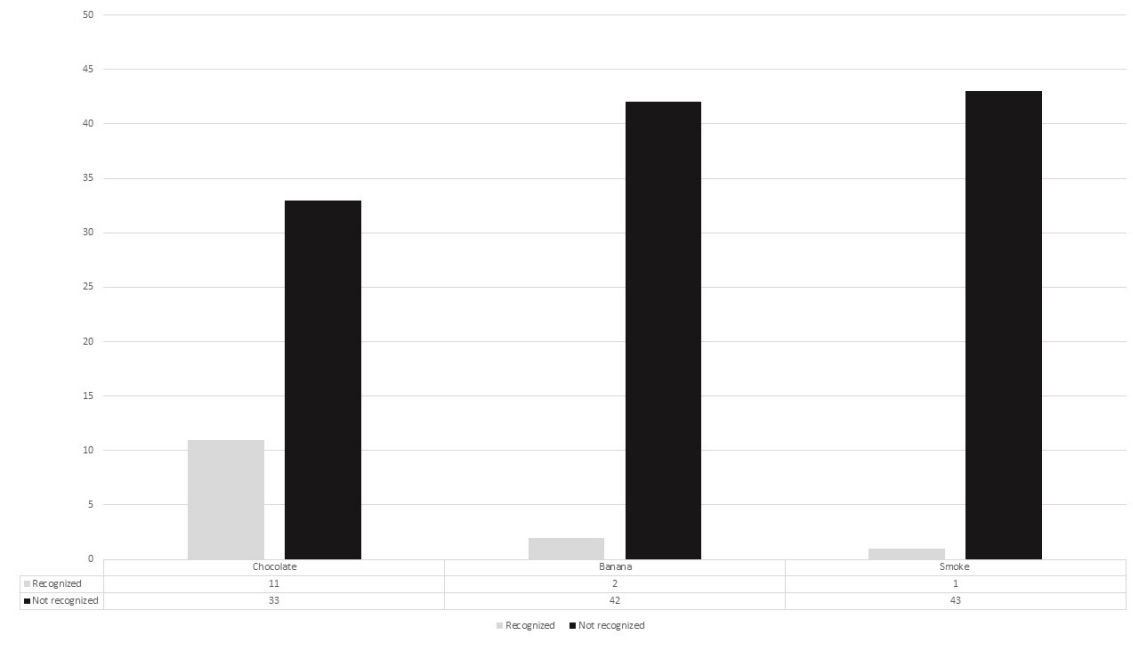

